\title{
Plasma Fibronectin Synthesis in Normal and Injured Humans as Determined by Stable Isotope Incorporation
}

\author{
Claudia Thompson, Frank A. Blumenstock, Thomas M. Saba, Paul J. Feustel, \\ John E. Kaplan, John B. Fortune, Lindsay Hough, and Vera Gray \\ Departments of Biochemistry, Physiology, Surgery, and Pharmacology, Albany Medical College, Albany, New York 12208
}

\begin{abstract}
In humans, plasma fibronectin decreases early after operative injury, burn, or trauma, followed by a rapid restoration with a secondary decline typically observed if such patients become septic. We determined the rate of plasma fibronectin and plasma fibrinogen synthesis in normal subjects and injured patients using a stable isotope incorporation technique with $\left[{ }^{15} \mathrm{~N}\right] \mathrm{glycine}$. During a constant 14-h infusion of $\left[{ }^{15} \mathrm{~N}\right] \mathrm{glycine}$, the enrichment of $\left[{ }^{15} \mathrm{~N}\right] \mathrm{glycine}$ in both the free plasma glycine precursor pool as well as the urinary hippurate pool was determined; the latter used as an estimate of intracellular hepatic precursor enrichment. $\left[{ }^{15} \mathrm{~N}\right] \mathrm{Glycine}$ enrichment in both plasma fibronectin and fibrinogen was also quantified. The synthesis rate $\left(J_{s} / V\right)$ expressed in micrograms per milliliter of plasma per hour and the fractional synthesis rate (FSR) expressed as percentage of the plasma pool produced per day were determined. In normal subjects, the FSR for plasma fibronectin using ${ }^{15} \mathrm{~N}$ enrichment into urinary hippurate was 35.35 $\pm 1.46 \% / \mathrm{d}$, whereas the $J s / V$ was $4.45 \pm 0.19 \mu \mathrm{g} / \mathrm{ml}$ plasma per h. In normal subjects, the FSR for plasma fibronectin using ${ }^{15} \mathrm{~N}$ enrichment into free plasma glycine was $14.73 \pm 0.63 \% / \mathrm{d}$, whereas the $J_{s} / V$ was $1.98 \pm 0.09 \mu \mathrm{g} / \mathrm{ml}$ plasma per $h$. Early (2-3 d) after burn injury, fibronectin synthesis was increased $(J s / V=5.74 \pm 0.36 ; P<0.05)$, whereas later after injury, fibronectin synthesis began to decline $(J s / V=3.52 \pm 0.24 ; P$ $<0.05$ ) based on ${ }^{15} \mathrm{~N}$ enrichment of urinary hippurate. In contrast, the $J_{s} / V$ and FSR of plasma fibrinogen, a well-documented acute-phase plasma protein, revealed a sustained elevation $(P<0.05)$ after injury in both the trauma and burn patients. Thus, plasma fibronectin synthesis is elevated early postinjury, which may contribute to the rapid restoration of its blood level. However, once fibronectin levels have normalized, the synthesis of plasma fibronectin appears to decline.
\end{abstract}

\section{Introduction}

Fibronectin is a high molecular mass dimeric glycoprotein $\left(M_{\mathrm{r}}\right.$ $450 \mathrm{kD})$ that circulates in plasma at a concentration of $350-450 \mu \mathrm{g} / \mathrm{ml}(1-3)$. It has a unique domain structure with a

Claudia Thompson is a predoctoral trainee in the Department of Biochemistry at Albany Medical College.

Reprint requests to Dr. Saba, Department of Physiology (A-134), Albany Medical College, 47 New Scotland Avenue, Albany, NY 12208. 1989.

Received for publication 7 July 1988 and in revised form 9 June

J. Clin. Invest.

(C) The American Society for Clinical Investigation, Inc.

0021-9738/89/10/1226/10 \$2.00

Volume 84, October 1989, 1226-1235 high affinity for collagen, actin, fibrin, Clq, and other molecules $(2,3)$. Plasma fibronectin mediates macrophage or reticuloendothelial phagocytic clearance of a variety of denatured or foreign particulate materials and Clq containing immune complexes, potentially in the blood after tissue injury, disseminated intravascular coagulation, or sepsis. Cellular or tissue fibronectin is a less soluble form of fibronectin (4), localized in the extracellular matrix of many cells and in connective tissue matrices (5). Cellular fibronectin influences cell-to-substratum adherence as well as cell-to-cell interactions and accordingly plays a role in tissue integrity $(2,3)$. Plasma fibronectin is antigenically related to cellular fibronectin but differs especially with respect to subunit structure and solubility (4). Plasma fibronectin can incorporate into the extracellular matrix as determined by fluorescent (6) and isotopic analysis (7), where it codistributes with endogenous tissue fibronectin. Such incorporation may influence endothelial adherence and lung vascular integrity (8).

RE phagocytic depression after trauma or burn occurs in association with low levels of plasma fibronectin $(3,9,10)$. In patients, plasma fibronectin deficiency most often develops early after trauma, burn, or major surgical intervention as well as during periods of severe sepsis postinjury in association with multiple organ failure $(3,11,12)$. Since the opsonic deficiency after trauma or burn can be reversed by infusion of fresh $(<2$ mo old) fibronectin-rich plasma cryoprecipitate (11-13) or purified intact human fibronectin (14), it has been suggested that infusion of opsonically active plasma fibronectin may provide an additional modality of therapy for septic injured patients. Although this remains to be documented in controlled clinical studies, it is apparent that such therapeutic approaches will require an understanding of the synthesis and turnover of plasma fibronectin.

We utilized a stable isotope infusion technique $(15,16)$ to determine the rate of plasma fibronectin synthesis in normal humans and injured patients. Emphasis was placed on evaluating this parameter early (2-3 d) after burn injury, in order to determine whether enhanced synthesis may contribute to its rapid plasma restoration often observed within 2-3 d after the initial postinjury depletion. Moreover, since in vitro findings (17) have suggested that plasma fibronectin may behave as an acute-phase plasma protein, its synthesis was compared to plasma fibrinogen, a well-documented acute-phase protein.

\section{Methods}

\section{Experimental design}

The protocol was approved by the Human Studies Committee, and informed consent was obtained from each subject. Each participant received a physical examination, including blood chemistry analysis, hematological analysis, and urinalysis, before entry into the protocol. For healthy subjects, after admission to Albany Medical Center Hospital, an intravenous line was placed in each arm: one for the infusion of 
$\left[{ }^{15} \mathrm{~N}\right]$ glycine ( $\geq 99 \%$ purity; Isotech Inc., Dayton, $\mathrm{OH}$ ) and the other for obtaining serial blood samples. For all studies, baseline blood and urine samples were taken before initiation of glycine infusion. The stock $\left[{ }^{15} \mathrm{~N}\right]$ glycine was prepared in $0.9 \% \mathrm{NaCl}$ at a concentration of 50 $\mathrm{mg} / \mathrm{ml}$, sterilized by filter, and tested for pyrogenicity before use by Leberco Testing, Inc., Roselle Park, NJ. The infusate containing $\left[{ }^{15} \mathrm{~N}\right]$ glycine was prepared in $0.45 \% \mathrm{NaCl}$ and $5.0 \%$ glucose. Each subject received $2.5 \mathrm{mg}$ of $\left[{ }^{15} \mathrm{~N}\right]$ glycine $/ \mathrm{kg}$ per $\mathrm{h}$ at a constant infusion flow rate of $30 \mathrm{ml} / \mathrm{h}$. Before beginning the infusion of $\left[{ }^{15} \mathrm{~N}\right]$ glycine, each subject received a $10-\mathrm{ml}$ priming dose of $1.0 \mathrm{mg} / \mathrm{kg}\left[{ }^{15} \mathrm{~N}\right]$ glycine. The $\left[{ }^{15} \mathrm{~N}\right]$ glycine infusion lasted for $14 \mathrm{~h}$ during which time blood and urine samples were obtained at hourly intervals. Blood $(15 \mathrm{ml})$ was collected into tubes containing EDTA (disodium salt) to prevent coagulation. Plasma collected immediately after centrifugation of the blood was utilized for purification of fibronectin, fibrinogen and plasma amino acids.

\section{Isolation of plasma fibronectin and its hydrolysis}

Fibronectin was isolated from plasma by gelatin-Sepharose affinity chromatography as previously described $(7,8,10)$. Samples $(0.1 \mathrm{ml})$ of the eluted fibronectin were analyzed for fibronectin purity by SDSPAGE analysis. Trichloroacetic acid (TCA) was added (25\% vol/vol) to the fibronectin and the mixture was iced for $30 \mathrm{~min}$ to precipitate the fibronectin. The tubes were centrifuged for $10 \mathrm{~min}$ at $2,000 \mathrm{rpm}$ and the supernatant was discarded. The precipitated fibronectin was washed with $2.0 \mathrm{ml}$ of $95 \%$ ethanol and centrifuged, and the ethanol wash was discarded. The residual ethanol was removed by evaporation to dryness in a concentrator (Speed Vac, Savant Instruments, Inc., Farmingdale, NY). When dry, $1.0 \mathrm{ml}$ of $6 \mathrm{~N} \mathrm{HCl}$ was added and the samples were gassed with nitrogen and then heated for $18 \mathrm{~h}$ at $110^{\circ} \mathrm{C}$ to hydrolyze the purified fibronectin to its constituent amino acids. After hydrolysis, the $\mathrm{HCl}$ was evaporated and the dry residue containing amino acids from each sample was derivatized to $N$-acetyl- $C$-propyl derivatives for analysis by gas chromatography-mass spectrometry (GC-MS)' (15).

\section{Isolation of fibrin and its hydrolysis}

Fibrin was isolated from plasma by clotting $0.5 \mathrm{ml}$ of fibronectin-deficient plasma with $5 \mathrm{U}$ of human thrombin (courtesy of Dr. John Fenton, Wadsworth Research Laboratories, New York State Health Department). Fibrin was isolated rather than purifying fibrinogen from plasma, since the enrichment of the precursor glycine into fibrin should be identical to its enrichment into fibrinogen. The samples were allowed to clot at room temperature for $18 \mathrm{~h}$. The fibrin clot was collected by alternately microcentrifuging and washing with $0.015 \mathrm{M}$ tosyl-arginine methyl ester. SDS-PAGE analysis of the clot under reducing conditions was performed to validate the specificity of the purification procedure. It demonstrated three major bands at 65,54 , and $48 \mathrm{kD}$ corresponding respectively to the well-documented $\alpha-, \beta$-, and $\gamma$-chains of fibrin. Hydrolysis of fibrin into its amino acid constituents and preparation of derivatives for GC-MS analysis were performed as described above for fibronectin.

\section{Isolation of plasma amino acids}

Isolation and derivatization of amino acids were performed as described by Wolfe (15). Ion exchange columns (Bio-Rad Laboratories, Richmond, CA), used for the isolation of plasma amino acids, were obtained as the econo-columns prefilled with Dowex AG 50W-X8 (H+ form; Dow Corning Corp., Midland, MI). $1 \mathrm{ml}$ of plasma was used to determine the enrichment of $\left[{ }^{15} \mathrm{~N}\right]$ glycine into the plasma amino acid precursor pool. To this plasma, $1 \mathrm{ml}$ of $10 \%$ TCA was added, vortexed, and allowed to stand on ice for $30 \mathrm{~min}$. The suspension was centrifuged for $10 \mathrm{~min}$ at $2,000 \mathrm{rpm} .1 \mathrm{ml}$ of the supernatant was diluted with $10 \mathrm{ml}$ of distilled water and applied to an ion ex-

1. Abbreviations used in this paper: APE, atoms percent excess; FSR, fractional synthesis rate; GC-MS, gas chromatography/mass spectrometry. change econo-column preconditioned with distilled water so that the $\mathrm{pH}$ of the water going into the columns was identical to that eluting from the columns. After application of the diluted TCA supernatant solution, the columns were washed extensively with distilled water. The amino acids were eluted from the columns with $2 \mathrm{M}$ ammonium hydroxide. The first $1.5 \mathrm{ml}$ of the ammonium hydroxide wash was discarded while the next $3.5 \mathrm{ml}$ of the eluate was collected, evaporated to dryness, and stored for derivatization.

\section{Isolation of urinary hippurate}

Prefilled octadecyl $\left(\mathrm{C}_{18}\right)$ columns (J. T. Baker Chemical Co., Phillipsburg, NJ) for the isolation of urinary hippurate were washed with 3.0 $\mathrm{ml}$ of HPLC grade methanol followed by a $3.0-\mathrm{ml}$ wash with distilled water. A 1-ml sample of urine that had been acidified by the addition of $0.1 \cdot \mathrm{ml}$ of $1 \mathrm{~N} \mathrm{HCl}$ was applied to the $C_{18}$ columns. The columns were washed with $3.0 \mathrm{ml}$ of distilled water and the bound hippuric acid was eluted with $5.0 \mathrm{ml}$ of acetonitrile. The acetonitrile was removed by evaporation and the dry residue was stored for subsequent derivatization.

\section{Derivatization of amino acids}

Reagents for the preparation of the $\mathrm{N}$-acetyl- $\mathrm{C}$-propyl derivatives of amino acids for GC-MS analysis were purchased from Alltech Associates, Deerfield, IL. To all amino acid samples (standards, protein hydrolysates, plasma amino acids) $50 \mu \mathrm{l}$ of the water scavenger, 2,3-dimethoxypropane, was added followed by the addition of $2 \mathrm{ml}$ of the esterification reagent ( $3.5 \mathrm{~N} \mathrm{HBr}$ in propanol). The reaction tubes (16 $\times 100$-mm screw cap tubes with teflon-lined tops) were flushed with nitrogen, capped, and heated at $110^{\circ} \mathrm{C}$ for $20 \mathrm{~min}$. The propanol- $\mathrm{HBr}$ reagent was removed using the Speed Vac concentrator while heating to $45^{\circ} \mathrm{C}$. After apparent dryness, $2.0 \mathrm{ml}$ of an azeotropic solution of $N$-propanol/benzene (30:70) was added and the samples were again evaporated to dryness. After the samples were cooled to room temperature, the amino-acid ester salt was combined with $2.0 \mathrm{ml}$ of the acetylation reagent ( $1 \mathrm{vol}$ acetic anhydride/ $2 \mathrm{vol}$ of triethylamine $/ 5 \mathrm{vol}$ of acetone). The reaction tubes were flushed with nitrogen and heated at $60^{\circ} \mathrm{C}$ for $3 \mathrm{~min}$. The solution was evaporated to dryness at $60^{\circ} \mathrm{C}$ under a slow stream of nitrogen. Another $2.0 \mathrm{ml}$ of azeotrope was added and each sample was evaporated. The oily residue in the tubes was dissolved in $2.0 \mathrm{ml}$ of ethyl acetate and $1 \mathrm{ml}$ of saturated $\mathrm{NaCl}$ was added. The tubes were then shaken vigorously and allowed to stand until two well-defined layers were formed. A $0.750-\mathrm{ml}$ aliquot of the top layer was transferred to new tubes and the ethyl acetate was evaporated under a slow stream of nitrogen. After drying, the residue was redissolved in $1.0 \mathrm{ml}$ of ethyl acetate and aliquots were taken for analysis by GC-MS. Standards for the GC-MS analysis consisted of defined concentrations of $\left[{ }^{15} \mathrm{~N}\right] \mathrm{glycine}$ and $\left[{ }^{14} \mathrm{~N}\right]$ glycine. These standards ranged from $0 \%$ to $20 \%\left[{ }^{15} \mathrm{~N}\right] \mathrm{glycine}$.

\section{Derivatization of urinary hippuric acid}

To derivatize the urinary hippurate, we used $N$-methyl- $N$-(tertbutyldimethylsilyl)-trifluoroacetamide (MTBSTFA; Pierce Chemical Co., Rockford, IL). A $200-\mu l$ volume of silylation grade acetonitrile was added to the dried hippuric acid as well as to $\left[{ }^{15} \mathrm{~N}\right]$ hippurate and $\left[{ }^{14} \mathrm{~N}\right]$ hippurate standards. This was combined with $200 \mu \mathrm{l}$ of the derivitizing reagent. The reaction tubes were flushed with nitrogen and allowed to react at $70^{\circ} \mathrm{C}$ for $60 \mathrm{~min}$ before GC-MS analysis.

\section{GC-MS analysis}

To determine the isotopic abundance (16) in the derivatized samples, a gas chromatograph (model 5890, Hewlett-Packard Co., Palo Alto, CA) equipped with an electron impact mass spectrometer detector (model 5870, Hewlett-Packard Co.) was used. The GC column (Ultra, Hewlett-Packard Co.) was $5 \%$ phenylmethyl silicone with a $0.33-\mu \mathrm{m}$ film thickness and $0.2-\mathrm{mm}$ internal diameter. The system included a data processor (model 9836C, Hewlett-Packard Co.). For separation of the $N$-acetyl- $C$-propyl derivatives of amino acids, the $\mathrm{GC}$ conditions were injector port temperature of $190^{\circ} \mathrm{C}$, transfer line at $250^{\circ} \mathrm{C}$, helium 
carrier gas flow rate of $60 \mathrm{ml} / \mathrm{min}$ with a back pressure of $16 \mathrm{psi}$, and an initial oven temperature of $60^{\circ} \mathrm{C}$ for $2 \mathrm{~min}$ followed by a programmed temperature increase of $35^{\circ} \mathrm{C} / \mathrm{min}$ until $240^{\circ} \mathrm{C}$. The selected ions for calculation of glycine enrichment were $119 / 118 \mathrm{~m} / \mathrm{z}$ ratio and the confirming ion pair was $101 / 100 \mathrm{~m} / \mathrm{z}$ with a retention time of $\sim 6.5$ min. For analysis of the hippuric acid derivative, all conditions were identical except that the initial oven temperature was $120^{\circ} \mathrm{C}$ for $1 \mathrm{~min}$ followed by a programmed temperature increase of $70^{\circ} \mathrm{C} / \mathrm{min}$ to $240^{\circ} \mathrm{C}$. The selected ions were $237 / 236 \mathrm{~m} / \mathrm{z}$ ratio for calculation of enrichment and $193 / 192 \mathrm{~m} / \mathrm{z}$ for confirming ions with a retention time of $\sim 7.5 \mathrm{~min}$.

\section{Analysis of plasma fibronectin levels}

Plasma fibronectin was measured over the 14-h protocol by immunoturbidimetric analysis (Boehringer Mannheim Diagnostics, Houston, TX) using a bichromatic analyzer (model ABA-100, Abbott Laboratories, Irving, TX) as described (1).

\section{Analysis of plasma fibrinogen levels}

Plasma fibrinogen was measured by a modified Mancini radial immunodiffusion assay (18), using goat anti-human fibrinogen antibody (Cappel Organo Teknika Corp., West Chester, PA). The precipitin ring diameter was quantified by comparison to standard curves using purified fibrinogen.

\section{Determination of the synthesis rate of fibronectin}

Using the ion ratios determined by GC-MS, the atoms percent excess (APE) of the $\left[{ }^{15} \mathrm{~N}\right]$ glycine precursor in plasma fibronectin, plasma fibrinogen, plasma amino acids, and urinary hippurate was calculated by the method of Millard (16). These data were used in the compartmental model presented in Fig. 1, which is the same for either fibronectin or fibrinogen. The model will be described in detail because it has not been previously documented, especially with respect to the synthesis of specific plasma proteins in humans such as fibronectin or fibrinogen.

Definitions for the model in Fig. $1 . S(t)$ is the APE of $\left[{ }^{15} \mathrm{~N}\right] \mathrm{glycine}$ in the fibronectin or fibrinogen pool as a function of time. Si $(t)$ is the APE of $\left[{ }^{15} \mathrm{~N}\right]$ in the precursor pool. Since the APE of $\left[{ }^{15} \mathrm{~N}\right] \mathrm{glycine-tRNA}$ in hepatocytes cannot be directly measured, two different precursor enrichments were used to separately approximate the intracellular enrichment. The first was the plasma $\left[{ }^{15} \mathrm{~N}\right]$ glycine enrichment. Since plasma fibronectin (or plasma fibrinogen) is synthesized and released into the plasma from liver hepatocytes $(19,20)$, the second was the APE of urinary hippurate which may be considered to be equivalent to the intracellular enrichment $(21,22)$. For completeness, we utilized both approaches, i.e., with $S i(t)$ equal to the plasma glycine APE, and with $S i(t)$ equal to the urinary hippurate APE to calculate the endogenous rate of synthesis of plasma fibronectin and fibrinogen. With reference to the model depicted in Fig. 1, the following explanation is provided.

$C(t)$ is the concentration of fibronectin (or fibrinogen) as a function of time in the plasma pool, expressed as micrograms per milliliter. $V$ is the volume in milliliters of the compartment through which fibronectin (or fibrinogen) is distributed at the concentration $C . J d$ is the disappearance rate of fibronectin (or fibrinogen) from the pool, expressed as micrograms per hour. $J s$ is the flux of fibronectin (or fibrinogen) into the plasma fibronectin (or fibrinogen) pool or the rate of

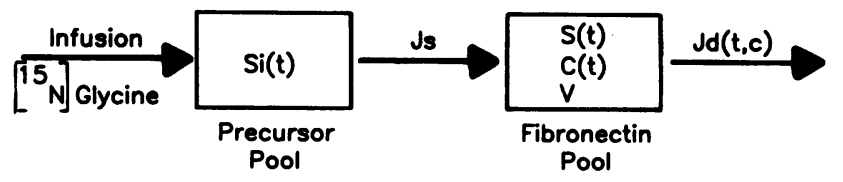

Figure 1. Compartmental model used in the data analysis and calculation of the synthetic rate of plasma fibronectin. The analogous model was used for analysis of the fibrinogen synthesis plasma fibronectin (or fibrinogen) synthesis, expressed as micrograms per hour.

Assumptions for the model presented in Fig. 1. (a) Jd is assumed to take place from a well-mixed compartment. $J d$ is not constrained to be constant and it may be a function of time and/or $C$. (b) $J s$ is assumed to be constant over the 14-h experimental protocol. $J s$, which is the rate of synthesis of fibronectin (or fibrinogen) is assumed to be exclusively from a well-mixed tracer precursor pool. (c) $V$, which is the fibronectin (or fibrinogen) distribution space, is assumed to be constant over the 14-h protocol. Because the size of the compartment $V$ cannot be measured directly in humans, $J s / V$ was estimated directly from the data. Although unlikely in normal individuals, it is possible that differences in $J S / V$ between subjects could be due to differences in the size of the fibronectin (or fibrinogen) distribution space, rather than differences in actual rate of fibronectin (or fibrinogen) synthesis. However, the calculation of $J s / V$ as an index of synthesis rate, instead of the absolute $J s$ value alone, normalizes the estimate to fibronectin (or fibrinogen) pool volume and thus minimizes individual differences resulting from body size differences, which although small, could not be controlled in this clinical study.

As mathematically demonstrated below, the value of $J d$ has no influence on the synthesis rate determination except as it affects $C(t)$. Since $C(t)$ was directly measured and incorporated into the regression, it was not necessary to measure or determine $J d$ in order to determine $J s$. Moreover, if there was no consistent change in $C(t)$ over the 14-h duration of the protocol, then it would follow that $J d=J s$. By applying the conservation of mass principle to fibronectin (or fibrinogen), we find that:

$\mathrm{d} C V / \mathrm{d} t=J s-J d$.

By applying the conservation of mass principle to $\left[{ }^{15} \mathrm{~N}\right]$ glycine, we find that:

$\mathrm{d}(S C V) / \mathrm{d} t=J s S i-J d S$.

By taking the derivative (assuming $V$ to be constant) coupled with substituting and isolating the time derivative, we find that:

$C V(\mathrm{~d} S / \mathrm{d} t)+S V(\mathrm{~d} C / \mathrm{d} t)=J s S i-J d S$

or

$\mathrm{d} S / \mathrm{d} t=[(J s / V)(1 / C)][(S i-S)]$.

\section{Numerical integration and nonlinear regression}

Eq. 3b was directly fit to the data by numerical integration and nonlinear regression. The values of $S i(t), C(t)$, and $S(t)$ were directly measured. The value of $J s / V$ and its asymptotic standard deviation were determined from these data by a nonlinear regression routine BMDPAR (23), which includes a numerical integrator to fit differential equations (24). The values of $\mathrm{Si}$ for each time point were taken to be either plasma glycine or urinary hippurate enrichment with the trace isotope, i.e., $\left[{ }^{15} \mathrm{~N}\right]$ glycine.

Using the technique described above, it is apparent that one advantage is that it is not necessary to consider the precursor enrichment, $S i$, or the fibronectin concentration, $C$, to be either constant, a step function or, indeed, any specific analytic function of time. A further advantage is that asymptotic standard deviations are calculated which reflect errors in measurements of all system variables rather than just the error in the measurement of fibronectin (or fibrinogen) enrichment.

Conversely, instead of considering the volume of distribution of fibronectin (or fibrinogen), $V$, to be constant, the total fibronectin (or fibrinogen) pool, $C V$, can also be assumed constant over the 14-h protocol. This alternative assumption appears justified, especially in normal subjects since there was little change in $C$ (fibronectin and fibrinogen concentration) with time and there is no reason to suspect that $V$ would change. Thus, $J s / V C$ can be determined without regard to the fibronectin (or fibrinogen) concentration. Since the expression $J s / V C$ is also referred to in the literature $(25)$ as the fractional synthesis 
rate (FSR), the data obtained in the current study was also expressed using this terminology. The expressions $\tau$ and $T_{1 / 2}$ are also used to describe these synthesis rates. $\tau$ is the time it takes for the product enrichment to reach $63 \%$ of the precursor enrichment and is calculated by taking the reciprocal of the respective FSR. $T_{t}$, half-time, is calculated by multiplying $\tau$ by 0.693 , which is the natural $\log$ of $2(\ln 2)$.

Brief case histories of healthy and injured patients studied

The healthy volunteer subjects consisted of nine individuals (22-44 yr old) who were not on medication which would be expected to alter their rate of protein synthesis. For the injured patients, we selected those patients who were at least 2-3 d or more postinjury, in order to avoid the early postinjury phase of acute depletion of plasma fibronectin $(12,26,27)$. Thus, by $2-3 \mathrm{~d}$ a rebound restoration has typically taken place. The injured patients were as follows. Patient 10 (ES) was a 55 -yr-old woman who sustained a $45 \%$ body surface burn with second-degree burns on her face, scalp, back, and extremities and thirddegree burns on her shoulders, back, and extremities. Her plasma fibronectin at the zero time of study was $304 \mu \mathrm{g} / \mathrm{ml}$. Patient 11 (FB) was a 40-yr-old man who was in a motor vehicle accident and sustained third-degree burns to the face and hands, second- and third-degree burns over the back, abdomen, and legs, as well as inhalation injury. His plasma fibronectin at the zero time of study was $294 \mu \mathrm{g} / \mathrm{ml}$. Patient 12 (MM) was a 66-yr-old woman who sustained $35 \%$ third-degree burns over right upper and lower extremities. Her plasma fibronectin at the zero time of study was $373 \mu \mathrm{g} / \mathrm{ml}$. Patient 13 (ES) was a 25-yr-old woman involved in a motor vehicle accident who sustained a head injury and massive chest injury. She had multiple rib fractures, hemo/pneumothorax, splenic rupture, left pulmonary contusion, and respiratory insufficiency. Her plasma fibronectin at the zero time of study was $235 \mu \mathrm{g} / \mathrm{ml}$, which was in the low end of normal, but it was relatively constant. Patient 14 (FB) was a 57-yr-old man who was involved in a motor vehicle accident and sustained an infraorbital fracture as well as pelvic and limb fractures. His plasma fibronectin at the zero time of study was $349 \mu \mathrm{g} / \mathrm{ml}$. Patient 15 (TL) was a 27-yr-old man who was quadraplegic due to a motor vehicle accident in which he sustained a pedicle and lamina fracture of the C-5 vertebra, as well as left acetabular fracture; $1 \mathrm{~d}$ before study, he underwent placement of a gastrostomy tube, tracheostomy, and fixation/stabilization of the C-5 and C-6 vertebrae. His plasma fibronectin at the zero time of study was $298 \mu \mathrm{g} / \mathrm{ml}$.

\section{Results}

To provide a composite analysis of the temporal enrichment response observed in the normal subjects, the averaged hourly enrichment of $\left[{ }^{15} \mathrm{~N}\right]$ glycine (expressed in APE) in plasma glycine, urinary hippurate, plasma fibronectin, and plasma fibrinogen is plotted in Fig. 2. Enrichment of $\left[{ }^{15} \mathrm{~N}\right]$ glycine into the plasma glycine pool over the 14-h protocol in normal subjects demonstrated a relatively consistent pattern, i.e., a marked early elevation in the enrichment followed by a further gradual increase. The temporal incorporation of $\left[{ }^{15} \mathrm{~N}\right]$ glycine into urinary hippurate in normal subjects revealed that average steady-state enrichment was achieved within $2 \mathrm{~h}$ after the

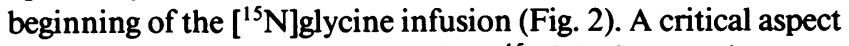
of this study was the rate at which $\left[{ }^{15} \mathrm{~N}\right] \mathrm{glycine}$ was incorporated into fibronectin or fibrinogen in plasma. Enrichment of the amino acid hydrolysates of plasma fibronectin or plasma fibrinogen occurred in all normal subjects, although the degree of enrichment was variable. Thus, in accordance with the model, we observed enrichment to begin with the plasma glycine pool, followed by enrichment of the intracellular hepatic glycine pool, with subsequent enrichment in both circulating fibronectin and fibrinogen (Fig. 2). Moreover, the enrichment of $\left[{ }^{15} \mathrm{~N}\right]$ glycine into urinary hippurate was only $\sim 50 \%$ of its

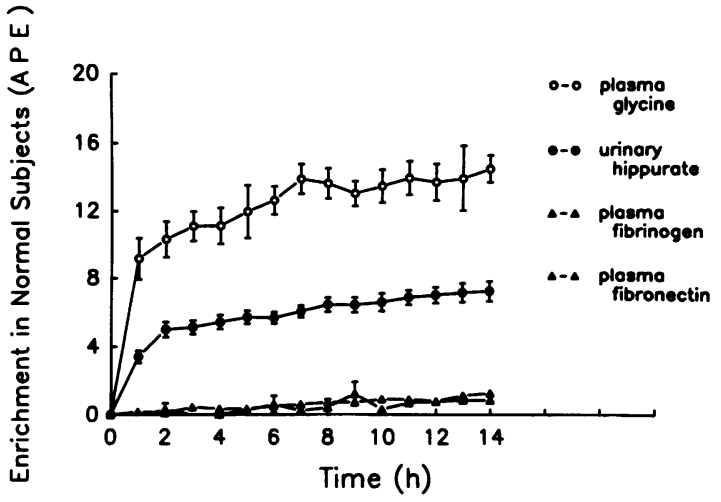

Figure 2. Averaged hourly enrichment of $\left[{ }^{15} \mathrm{~N}\right]$ glycine into plasma glycine, urinary hippurate, plasma fibronectin, and plasma fibrinogen over the 14-h protocol in normal subjects. Data are presented as mean \pm SE. Enrichment is expressed in terms of APE.

enrichment into the plasma precursor pool. This difference was expected and indirectly validates the model used, since there can be other sources of unlabeled intracellular hepatic glycine in addition to the glycine in plasma which can be incorporated into newly synthesized fibronectin from the liver.

The composite temporal enrichment observed in burn and trauma patients is presented in Figs. 3 and 4, respectively. The pattern of enrichment in burn patients (Fig. 3) was analogous to that observed in normal subjects, in that the enrichment into urinary hippurate was $\sim 50 \%$ less than that of plasma glycine. However, the magnitude of enrichment into both plasma glycine or urinary hippurate of burn patients was greater than normal subjects. The pattern of enrichment in the trauma patients, most of whom were studied at later intervals after injury is presented in Fig. 4. The ratio of enrichment between plasma glycine and urinary hippurate increased and approached 1. Although the magnitude of ${ }^{15} \mathrm{~N}$ enrichment in urinary hippurate was comparable to normal subjects, the enrichment in plasma glycine actually decreased in comparison to either normal subjects or burn patients.

The plasma concentration of fibronectin and fibrinogen was determined hourly over the $14-\mathrm{h}$ infusion period, and the

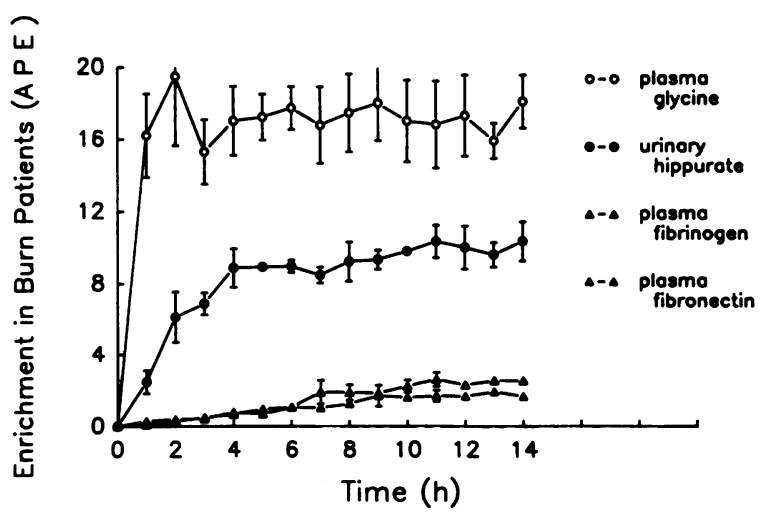

Figure 3. Averaged hourly enrichment of $\left[{ }^{15} \mathrm{~N}\right]$ glycine into plasma glycine, urinary hippurate, plasma fibronectin, and plasma fibrinogen over the 14-h protocol in burn patients $(n=3)$. Data are presented mean \pm SE. Enrichment is expressed in terms of APE. 


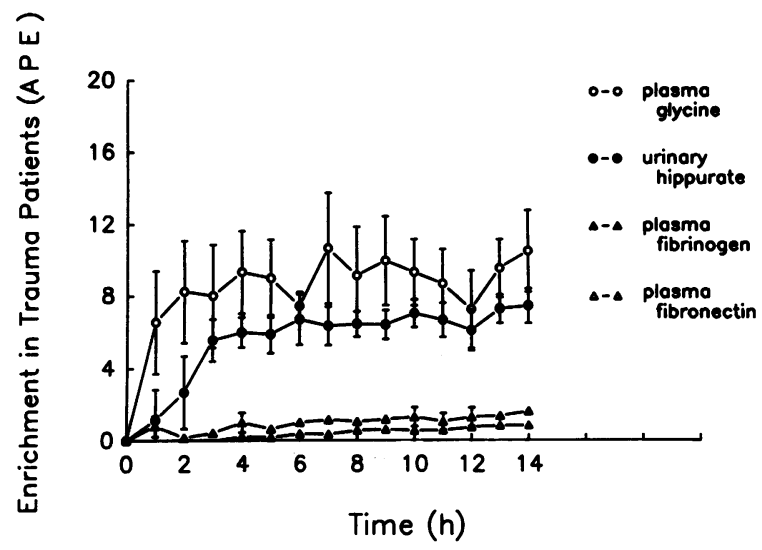

Figure 4. Averaged hourly enrichment of $\left[{ }^{15} \mathrm{~N}\right] \mathrm{glycine}$ into plasma glycine, urinary hippurate, plasma fibronectin, and plasma fibrinogen over the 14-h protocol in trauma patients $(n=3)$. Data are presented as mean $\mathbf{S E}$. Enrichment is expressed in terms of APE.

average level is presented in Tables I-IV. While not graphed on an hourly basis, the plasma concentration of both fibronectin and fibrinogen of each subject was essentially constant over the 14-h protocol. As expected, owing to the time interval postinjury, we observed no group difference (two-way ANOVA with repeated measures) in plasma fibronectin between injured patients and normal subjects, but plasma fibrinogen in injured patients was significantly elevated $(P<0.05)$ as expected (Tables III and IV).

Using the data presented in Figs. 2-4, plasma fibronectin and plasma fibrinogen synthesis rates $(J S / V$; FSR) were calculated (Tables I-IV) based on both plasma glycine enrichment
(Tables I and III) and urinary hippurate enrichment (Tables II and IV). As seen in Tables I and II, the rate of synthesis of plasma fibronectin in normal subjects is quite rapid. Based on plasma glycine as the precursor pool, the FSR in normal subjects ranged from 4.92 to $31.86 \% / \mathrm{d}$ with an average of $14.73 \pm 0.63 \% /$ d. The FSR in normal subjects using hippurate enrichment ranged from 9.14 to $49.87 \% / d$ with an average of $35.35 \pm 1.46 \% / \mathrm{d}$. Thus, based on urinary hippurate enrichment, which would be expected to closely reflect the synthesis of fibronectin by the liver, $\sim 35-36 \%$ of the plasma fibronectin pool is synthesized every day in normal adults. Early after burn injury (i.e., 2-3 d), the synthesis rate of fibronectin was increased (Tables III and IV), although later postinjury when fibronectin levels typically stabilized in these nonseptic posttrauma patients, the synthesis rate of fibronectin had actually declined or was normal. Variability in synthesis of fibronectin was consistent with the variable extent of injury in multiple trauma patients. In contrast, synthesis of fibrinogen was increased both early or late postinjury, consistent with its anticipated response as an acute-phase protein.

Quantitative analysis of data derived from urinary hippurate enrichment, which is the most precise estimate of the actual hepatic precursor pool obtainable in humans, reveals that the $T_{1 / 2}$ for plasma fibronectin in normal adults is $\sim 47 \mathrm{~h}$, whereas the $T_{1 / 2}$ for plasma fibrinogen in normal adults is $\sim 87 \mathrm{~h}$. In contrast, early after burn (2-3 d), the apparent $T_{1 / 2}$ for plasma fibronectin was $\sim 38 \mathrm{~h}$. Although the elevation of fibrinogen synthesis was sustained postinjury (Fig. 5), the increased synthesis of fibronectin was not sustained. Accordingly, based on urinary hippurate enrichment data, fibronectin synthesis was initially stimulated postinjury and then normalized (Fig. 6) as the concentrations of fibronectin was restored.

Table I. Parameters of Plasma Fibronectin and Plasma Fibrinogen Synthesis in Normal Subjects Using Plasma Glycine as the Precursor Pool

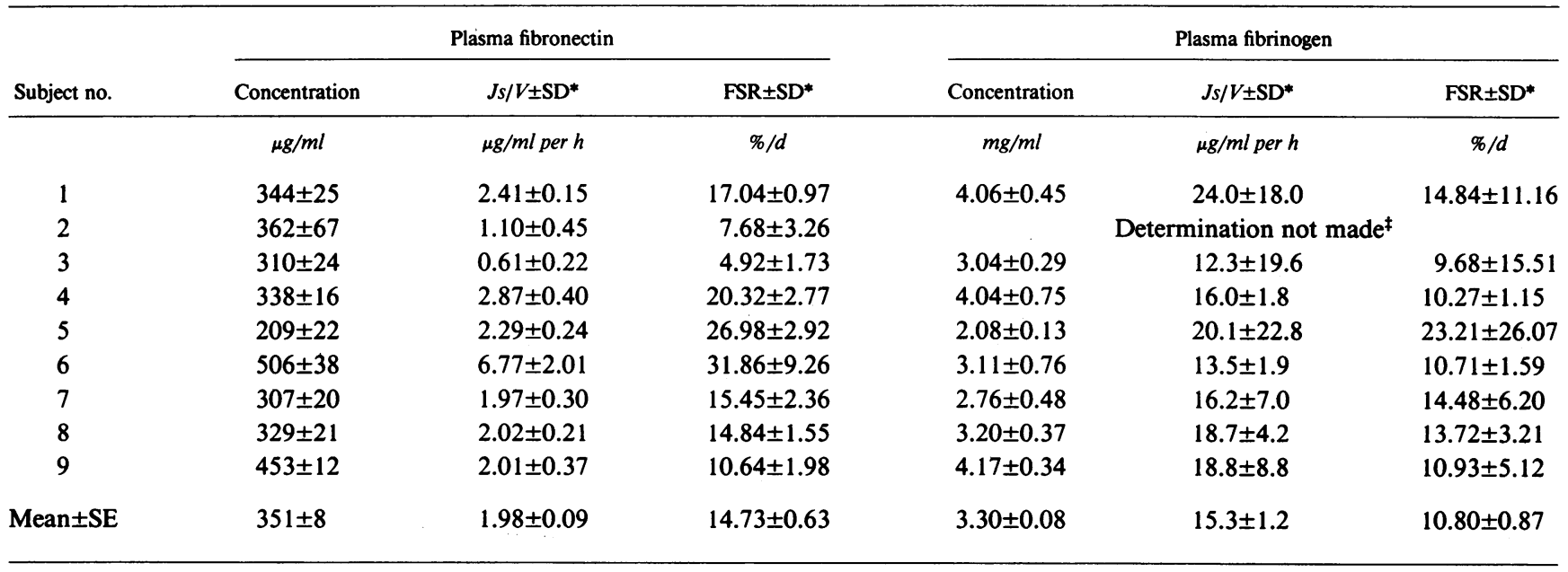

The means and standard errors for $J s / V$ and FSR are weighted based on the variance of the individual estimates. This method places more emphasis on values determined to have greater confidence, i.e., a smaller SD. For fibronectin, the unweighted mean $J s / V \pm \mathrm{SE}$ is $2.45 \pm 0.55 \mu \mathrm{g} / \mathrm{ml}$ per $\mathrm{h}$ and the unweighted mean FSR $\pm \mathrm{SE}$ is $16.64 \pm 2.74 \% / \mathrm{d}$. For fibrinogen, the unweighted mean $J s / V \pm \mathrm{SE}$ is $17.5 \pm 1.17 \mu \mathrm{g} / \mathrm{ml} \mathrm{per} \mathrm{h}$ and the unweighted mean FSR \pm SE is $13.48 \pm 1.04 \% / d$. Plasma concentrations reflect mean \pm SE over the 14-h protocol interval. * The synthesis rate $(J s / V)$ and the FSR were determined directly from the analysis of the enrichment data. The standard deviations of $J s / V$ and FSR are the calculated asymptotic standard deviations. The mean values of $\tau$ and $T_{1 / 2}$ derived from the respective values of FSR are 162.9 and $112.9 \mathrm{~h}$ for fibronectin, and 222.2 and $154.0 \mathrm{~h}$ for fibrinogen. ${ }^{\ddagger}$ Samples from subject 2 were inadvertently collected with heparin as opposed to using EDTA to prevent coagulation. The heparin interfered with fibrinogen quantitation and purification. Thus, plasma fibrinogen determinations were not made for subject 2. The large SD for $J s / V$ and FSR in several of the subjects reflects outlying data points for fibrinogen enrichment, entered into the regression for which no justified reason to exclude could be identified. 
Table II. Parameters of Plasma Fibronectin and Plasma Fibrinogen Synthesis in Normal Subjects Using Urinary Hippurate as the Precursor Pool

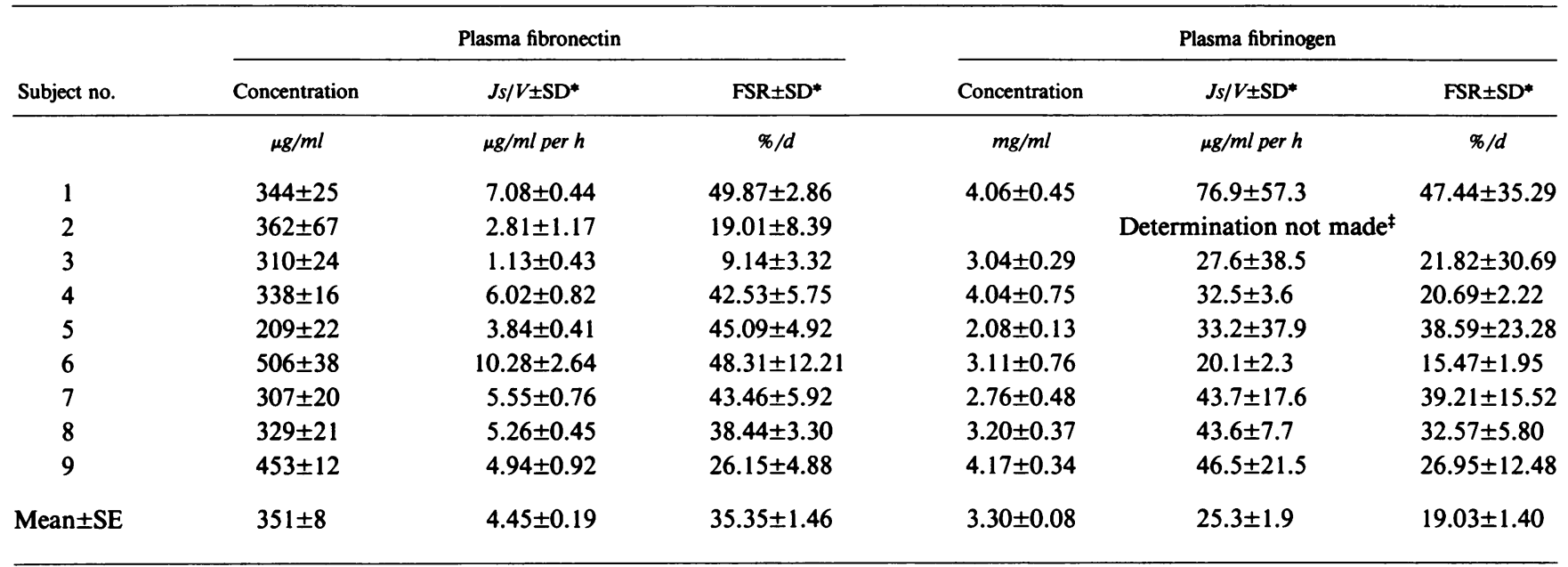

The means and standard errors for $J s / V$ and FSR are weighted based on the variance of the individual estimates. This method places more emphasis on values determined to have greater confidence, i.e., a smaller SD. For fibronectin, the unweighted mean $J s / V \pm \mathrm{SE}$ is $5.21 \pm 0.67 \mu \mathrm{g} / \mathrm{ml}$ per $\mathrm{h}$ and the unweighted mean FSR $\pm \mathrm{SE}$ is $35.78 \pm 4.49 \% / \mathrm{d}$. For fibrinogen, the unweighted mean $J s / V \pm \mathrm{SE}$ is $40.5 \pm 5.37 \mathrm{ug} / \mathrm{ml}$ per $\mathrm{h}$ and the unweighted mean FSR \pm SE is $30.34 \pm 3.42 \% /$ d. Plasma concentrations reflect mean \pm SE over the 14-h protocol interval. ${ }^{*}$ The synthesis rate $(J s / V)$ and the FSR were determined directly from the analysis of the enrichment data. The standard deviations of $J s / V$ and FSR are the calculated asymptotic standard deviations. The mean values of $\tau$ and $T_{1 / 2}$ derived from the respective value of FSR are 67.9 and $47.1 \mathrm{~h}$ for fibronectin, and 126.1 and $87.4 \mathrm{~h}$ for fibrinogen. ${ }^{\ddagger}$ Samples from subject 2 were inadvertently collected with heparin as opposed to using EDTA to prevent coagulation. The heparin interfered with fibrinogen quantitation and purification. Thus, plasma fibrinogen determinations were not made for subject 2. The large SD for $J s / V$ and FSR in several of the subjects reflects outlying data points for fibrinogen enrichment, entered into the regression, for which no justified reason to exclude could be identified.

\section{Discussion}

Most plasma proteins are synthesized by liver parenchymal cells $(19,20)$, which utilize both plasma- and cellular-derived amino acids as precursors for such exported proteins. We employed a sophisticated stable isotope infusion method to quantitate the synthesis rate of plasma fibronectin in healthy subjects and injured patients and to compare its synthesis to a well-documented acute phase protein, such as fibrinogen. Fibronectin and fibrinogen synthesis were compared because $(a)$ the primary site of synthesis of both proteins is the hepatocyte; (b) $\sim 10 \%$ of the amino acid residues in each protein is glycine; $(c)$ the method of isolating each protein from the plasma achieves good purity; $(d)$ the expected turnover rate of both proteins is short enough to allow for adequate incorporation of the stable isotope. within the 14-h infusion protocol; and (e) other investigators have suggested that hepatic stimulating factor (IL-6) may influence the synthesis of both proteins, implying that a similar response pattern might be anticipated (17).

Table III. Parameters of Plasma Fibronectin and Plasma Fibrinogen Synthesis in Burn and Trauma Patients Using Plasma Glycine as the Precursor Pool

\begin{tabular}{|c|c|c|c|c|c|c|c|}
\hline \multirow[b]{2}{*}{ Subject no. } & \multirow[b]{2}{*}{ Condition } & \multicolumn{3}{|c|}{ Plasma fibronectin } & \multicolumn{3}{|c|}{ Plasma fibrinogen } \\
\hline & & Concentration & $J s / V \pm \mathrm{SD}^{*}$ & $\mathrm{FSR} \pm \mathrm{SD}^{*}$ & Concentration & $J s / V \pm \mathrm{SD}^{*}$ & $\mathrm{FSR} \pm \mathrm{SD}^{*}$ \\
\hline & & $\mu g / m l$ & $\mu g / m l$ per $h$ & $\% / d$ & $m g / m l$ & $\mu g / m l p e r h$ & $\% / d$ \\
\hline 10 (ES) & Burn & $303 \pm 16$ & $2.39 \pm 0.37$ & $18.99 \pm 0.03$ & $4.74 \pm 0.55$ & $61.8 \pm 2.2$ & $31.09 \pm 1.26$ \\
\hline 11 (FB) & Burn & $300 \pm 8$ & $2.34 \pm 0.62$ & $18.69 \pm 4.96$ & $7.13 \pm 0.63$ & $115.8 \pm 8.0$ & $39.40 \pm 2.62$ \\
\hline $12(\mathrm{MM})$ & Burn & $333 \pm 18$ & $2.98 \pm 0.20$ & $21.62 \pm 1.44$ & $6.16 \pm 0.91$ & $34.6 \pm 9.1$ & $27.59 \pm 4.45$ \\
\hline $13(\mathrm{ES})$ & Trauma & $253 \pm 24$ & $1.30 \pm 0.24$ & $12.13 \pm 2.21$ & $5.58 \pm 0.80$ & $64.9 \pm 14.9$ & $28.32 \pm 5.89$ \\
\hline 14 (FB) & Trauma & $344 \pm 17$ & $2.39 \pm 0.20$ & $16.69 \pm 1.40$ & $7.68 \pm 0.80$ & $89.0 \pm 15.3$ & $28.50 \pm 4.87$ \\
\hline 15 (TL) & Trauma & $298 \pm 12$ & $5.43 \pm 0.77$ & $43.92 \pm 6.23$ & $4.47 \pm 0.44$ & $72.5 \pm 39.7$ & $41.75 \pm 19.83$ \\
\hline
\end{tabular}

* The synthesis rate $(J s / V)$ and the FSR were determined directly from the analysis of the enrichment data. The standard deviations of $J s / V$ and FSR are the calculated asymptotic standard deviations. The mean values of $\tau$ and $T_{1 / 2}$ derived from the respective values of FSR are 116.0 and $80.4 \mathrm{~h}$ for fibronectin in the burn patients, and 146.5 and $101.5 \mathrm{~h}$ for fibronectin in the trauma patients. The mean respective values of $\tau$ and $T_{1 / 2}$ derived from the respective values of FSR are 74.2 and $51.4 \mathrm{~h}$ for fibrinogen in burn patients, and 83.1 and $57.6 \mathrm{~h}$ for fibrinogen in trauma patients. Plasma concentrations reflect mean \pm SE over the $14-\mathrm{h}$ protocol interval. The identified fibronectin levels were the average over the $14 \mathrm{~h}$, as compared to the zero time level noted in the case histories. 
Table IV. Parameters of Plasma Fibronectin and Plasma Fibrinogen Synthesis in Burn and Trauma Patients Using Urinary Hippurate as the Precursor Pool

\begin{tabular}{|c|c|c|c|c|c|c|c|}
\hline \multirow{2}{*}{$\begin{array}{c}\text { Subject } \\
\text { no. }\end{array}$} & \multirow[b]{2}{*}{ Condition } & \multicolumn{3}{|c|}{ Plasma fibronectin } & \multicolumn{3}{|c|}{ Plasma fibrinogen } \\
\hline & & Concentration & $J s / V \pm \mathrm{SD}^{*}$ & $\mathrm{FSR} \pm \mathrm{SD}^{*}$ & Concentration & $J s / V \pm \mathrm{SD}^{*}$ & $\mathrm{FSR} \pm \mathrm{SD}^{*}$ \\
\hline & & $\mu g / m l$ & $\mu g / m l$ per $h$ & $\% / d$ & $\mathrm{mg} / \mathrm{ml}$ & $\mu g / m l$ per $h$ & $\% / d$ \\
\hline 10 & Burn & $303 \pm 16$ & $4.63 \pm 0.64$ & $36.47 \pm 5.12$ & $4.74 \pm 0.55$ & $113.6 \pm 3.0$ & $58.04 \pm 1.57$ \\
\hline 11 & Burn & $300 \pm 8$ & $4.88 \pm 1.35$ & $38.76 \pm 10.80$ & $7.13 \pm 0.63$ & $56.6 \pm 16.7$ & $86.91 \pm 5.57$ \\
\hline 12 & Burn & $333 \pm 18$ & $6.45 \pm 0.47$ & $47.08 \pm 3.31$ & $6.16 \pm 1.91$ & $72.1 \pm 20.8$ & $61.70 \pm 10.39$ \\
\hline 13 & Trauma & $253 \pm 24$ & $2.07 \pm 0.39$ & $19.35 \pm 3.55$ & $5.58 \pm 0.80$ & $115.5 \pm 27.0$ & $46.71 \pm 10.20$ \\
\hline 14 & Trauma & $344 \pm 17$ & $3.95 \pm 0.36$ & $27.62 \pm 2.44$ & $7.68 \pm 0.80$ & $150.1 \pm 27.0$ & $48.29 \pm 8.59$ \\
\hline 14 & Trauma & $298 \pm 12$ & $5.56 \pm 0.57$ & $44.92 \pm 4.56$ & $4.47 \pm 0.44$ & $62.5 \pm 31.2$ & $35.63 \pm 15.80$ \\
\hline
\end{tabular}

* The synthesis rate $(J S / V)$ and the FSR were determined directly from the analysis of the enrichment data. The standard deviations of $J s / V$ and FSR are the calculated asymptotic standard deviations. The mean values of $\tau$ and $T_{1 / 2}$ derived from the respective values of FSR are 55.0 and $38.1 \mathrm{~h}$ for fibronectin in burn patients, and 85.1 and $59.0 \mathrm{~h}$ for fibronectin in trauma patients. The mean values of $\tau$ and $T_{1 / 2}$ derived from the respective values of FSR are 39.9 and $27.6 \mathrm{~h}$ for fibrinogen in burn patients, and 52.32 and $36.26 \mathrm{~h}$ for fibrinogen in trauma patients. Plasma concentrations reflect mean \pm SE over the 14 -h protocol interval.

The concentration of plasma fibronectin is a balance between its rate of synthesis and its rate of removal from plasma. Many factors can affect this balance. For example, mild inflammation after low-dose endotoxin (28), and increased levels of $T_{4}(29,30)$ have been associated with elevation of plasma fibronectin, whereas starvation $(31,32)$, the early period after trauma $(9,10)$, as well as intravascular coagulation $(33,34)$ are associated with decreased plasma fibronectin. Severe sepsis, after trauma and burn, is often but not always associated with low plasma fibronectin levels; resolution of the postinjury septic episode is associated with normalization of fibronectin $(26,27)$. But infection alone in the absence of injury does not tend to lower the plasma fibronectin level. The human response to postoperative or posttrauma infection is not easily simulated in rodents, since the enhanced synthesis of plasma fibronectin during mild postoperative sepsis in rats can often exceed the enhanced consumption and actually main- tain a normal or elevated fibronectin level (35). Acute decline in plasma fibronectin early after trauma appears to be due to its consumption as an opsonin as well as its sequestration at sites of tissue injury owing to its high affinity for actin, fibrin, and collagen $(2,3,7,27,36)$. However, this acute decline is often followed by a rapid normalization of fibronectin within a few days $(3,9,12,27)$, even in the face of massive fluid resuscitation (27) which suggests enhanced synthesis. Starvation can also induce a decline in plasma fibronectin, and restoration of food intake results in rapid normalization of fibronectin $(31,32)$.

As opposed to utilizing the clearance of radiolabeled fibronectin from the plasma compartment to indirectly assess fibronectin synthesis rate, we utilized stable isotope infusion technology, which is more rigorous and safer in humans. It is also a direct measure of the synthesis rate, since degradation or removal of fibronectin from the plasma compartment will have

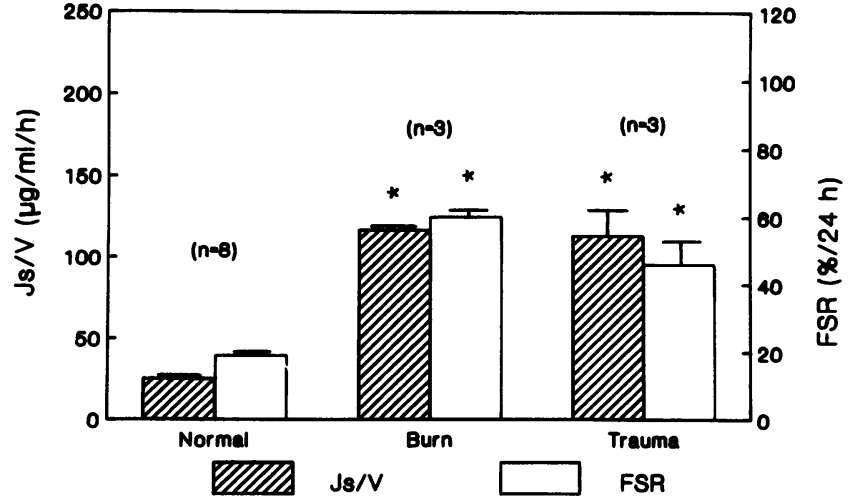

Figure 5. Plasma fibrinogen synthesis in normal subjects and injured patients based on ${ }^{15} \mathrm{~N}$ enrichment of the urinary hippurate pool. Values are weighted means $\pm \mathrm{SE}$ of the estimate. The $J_{S} / V$ is expressed as micrograms per milliliter per hour; the FSR is expressed as percent plasma pool per $24 \mathrm{~h}$. ${ }^{*}$ Differences $(P<0.05)$ reflect comparison with normal values. Student's $t$ test using Bonferoni's inequality for multiple comparisons was used.

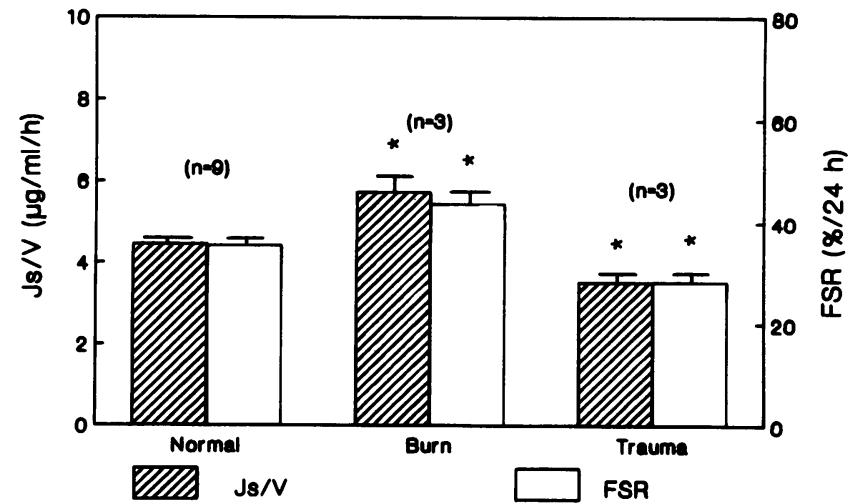

Figure 6. Plasma fibronectin synthesis in normal subjects and injured patients based on ${ }^{15} \mathrm{~N}$ enrichment of the urinary hippurate pool. The $J s / V$ is expressed as micrograms per milliliter per hour; the FSR is expressed as percent plasma pool per $24 \mathrm{~h}$. Values are weighted means \pm SE of the estimate. ${ }^{*}$ Differences $(P<0.05)$ expressed reflect comparison with normal values. Student's $t$ test using Bonferoni's inequality for multiple comparisons was used. 
no influence on the calculated rate. Synthesis determination is based upon the rate that fibronectin enrichment approaches precursor enrichment, and degradation will have no influence on this parameter. Moreover, rather than assessing total protein synthesis with $\left[{ }^{15} \mathrm{~N}\right]$ glycine incorporation as previously done $(21,22)$, we focused on measuring the synthetic rate of a particular plasma protein, i.e., plasma fibronectin or plasma fibrinogen. This analysis is appropriate for plasma fibronectin or plasma fibrinogen because their purification from plasma is readily achievable $(7,36)$ and $\left[{ }^{15} \mathrm{~N}\right]$ glycine enrichment into both proteins is quantifiable. Assuming that the newly synthesized $\left[{ }^{15} \mathrm{~N}\right]$ glycine-labeled proteins were purified to the same extent as the unlabeled plasma proteins, the extent of recovery is not a factor in our analysis. A high degree of purification was achieved for both fibronectin and fibrinogen.

The current study demonstrates that in adults, plasma fibronectin has a rapid synthetic and turnover rate, which can be further enhanced early after burn. Previously, Gersovitz et al. (37) as well as Yudkoff et al. (25) used stable isotope technology to study albumin synthesis in adults and premature infants, respectively. Also, Stein et al. (38) used this technology to study fibrinogen synthesis in adults. However, the current study documents for the first time the rate of synthesis of plasma fibronectin in healthy adults and injured patients using stable isotope technology. Using alternate methods, Sherman and Lee (33), utilizing exogenously labeled plasma fibronectin injected intravenously into rabbits observed a clearance $T_{1 / 2}$ of $71 \mathrm{~h}$. Azuma et al. (30) also obtained a clearance $T_{1 / 2}$ of 72-73 $\mathrm{h}$ in rabbits. Other studies in rats utilizing metabolically labeled fibronectin documented clearance $T_{1 / 2}$ 's of 10-28 h (7, 39). A normal FSR for fibronectin of $35-36 \% / d$, found in the current study based on enrichment of urinary hippurate, corresponds to a plasma $T_{1 / 2}$ of 45-50 h for fibronectin.

Our fibronectin $T_{1 / 2}$, based on the calculation of synthesis, differs substantially from the relatively rapid plasma clearance $T_{1 / 2}$ of $24 \mathrm{~h}$ obtained in normal humans by Pussell et al. (40) using prelabeled ${ }^{125}$ I-fibronectin. One difference could be that the exogenously labeled fibronectin, as used by Pussell et al. (40), was structurally altered so that its clearance was abnormally increased relative to normal fibronectin when injected in vivo. Sherman and Lee (33) presented data indicating preferential in vivo clearance of such iodinated fibronectin. Our rate of plasma fibronectin synthesis could be much lower than the true synthesis rate if the fibronectin purified from plasma was contaminated with slowly synthesized plasma proteins of relatively high glycine content. However, this is not likely, since PAGE demonstrated the homogeneity of the fibronectin isolated. Moreover, the method of isolating fibronectin from plasma by gelatin-Sepharose affinity chromatography is specific and efficient $(3,8,10,35,36)$. The possibility that the GC-MS analysis of the $\left[{ }^{15} \mathrm{~N}\right]$ glycine in the purified fibronectin was much less than reality is also not likely, since the GC-MS sensitivity is sufficient to measure enrichment of $\left[{ }^{15} \mathrm{~N}\right] \mathrm{glycine}$ accurately, even to a level of $0.5 \%$, and all the enrichments over $14 \mathrm{~h}$ were above this level. The possibility that the level of the precursor enrichment measured in plasma glycine or urinary hippurate was higher than actuality is also unlikely, since repeated analysis of standards revealed no discrepancies in the ion ratios. Therefore, the most likely reason for the difference between our findings based on stable isotope incorporation and those for humans $(40)$ or rabbits $(30,33)$ based only on clearance of iodinated fibronectin is either a preferential clearance of exogenously labeled fibronectin and/or a difference related to species, i.e., rabbits vs. humans. This conclusion is supported by the fact that despite the differences between literature values for the $T_{1 / 2}$ of fibronectin and that which we observed, the fibrinogen $T_{1 / 2}(87.4 \mathrm{~h})$ which we calculated based on urinary hippurate enrichment is in complete agreement with the $T_{1 / 2}$ of $81.6 \mathrm{~h}$ published by Stein et al. (38), who also used stable isotope infusion technology in humans.

In terms of species differences, Koj (41) highlighted the differences between human and rat synthesis rates with respect to $\mathrm{C}$-reactive protein, in that plasma $\mathrm{C}$-reactive protein rose dramatically in injured man and rabbits, but remained constant in rats. Conversely, $\alpha_{2}$-macroglobulin was much higher in rats after injury, but unaffected in humans after injury. In rats, the rate of plasma fibronectin synthesis appears to be very rapid even in the normal state (7) which is consistent with the ability of rats to maintain a high fibronectin level even in the presence of enhanced fibronectin consumption (35) and to normalize their fibronectin levels very rapidly after severe acute depletion posttrauma or endotoxemia $(9,28)$. Amrani et al. (17) suggested that fibronectin, like fibrinogen, may be an acute-phase protein in chickens, since the synthesis of both proteins is influenced, in part, by glucocorticoids and hepatic stimulator factor (IL-6). However, this concept is not supported by the data of Castell et al. (42), who recently reported that recombinant IL- 6 had a potent stimulating effect on the synthesis of acute-phase proteins such as fibrinogen and Creactive protein, but had no effect on fibronectin synthesis by isolated human hepatocytes.

In early models used to study acute-phase plasma protein synthesis, inflammation in animals was induced by injecting turpentine subcutaneously. Plasma proteins which increased dramatically after this stimulus were called acute-phase proteins; C-reactive protein and fibrinogen are classic examples. We observed no significant difference in the average plasma fibronectin between normal and injured patients at the time they were entered into our protocol, i.e., patients were studied beyond 2-3 d postinjury when fibronectin levels were expected to have normalized after the acute decline (27), if the patients did not become septic (3). However, plasma fibrinogen levels in the injured patients were approximately twofold higher than normal. This finding, coupled with the recent data by Castell et al. (42) that compared the effect of human IL-6 or so-called hepatic stimulating factor on both fibronectin and fibrinogen synthesis by human hepatocytes, suggests that fibronectin is not a classical acute-phase plasma protein like plasma fibrinogen in humans.

Thus, the current study documents for the first time with stable isotope technology that the synthesis of plasma fibronectin in adults is quite rapid with $\sim 35-36 \%$ of the plasma pool synthesized each day. Its corresponding $T_{1 / 2}$ in plasma is $47 \mathrm{~h}$ in normal adults. Early after burn injury when levels are rapidly normalizing, the fibronectin synthesis appears elevated. Accordingly, the effect of postinjury sepsis on fibronectin synthesis warrants future investigation, since this period of a more sustained deficiency in plasma fibronectin in humans $(11,12$, 14) may be linked to both enhanced consumption as well as impaired hepatic fibronectin synthesis. These patients are also the group of critically ill patients who develop multisystem organ failure with a high mortality rate $(43,44)$.

Note added in proof. The FSR as documented in the current study of normal and injured adults needs to be contrasted to the recent observations by Polin et al. (45) on fibronectin FSR in premature infants 
after $\left[{ }^{15} \mathrm{~N}\right]$ glycine infusion. In preterm neonates, they observed an FSR of $15.5 \pm 9.9 \%$ per day, which is $50-55 \%$ lower than our measured FSR in normal adults. This may be the basis for the lower plasma fibronectin in the newborns (45), even though its plasma clearance in newborns is slower than adults. Accordingly, both accelerated loss from plasma, especially in injured patients, as well as altered fibronectin synthesis can contribute to altered plasma fibronectin levels in humans.

\section{Acknowledgments}

The authors acknowledge Drs. Mollie McCormick and Michael Albert (Trauma Unit Fellows), Nancy Eberle, Linda Graca, and Julie Hasselbarth for technical assistance and Maureen Davis and Debbie Moran for manuscript preparation.

This study was supported primarily by the National Institutes of Health Trauma Center grant GM-15426 entitled "On-line Computer Therapy in Human Shock" and by GM-21447 entitled "Systemic Host Defense Following Trauma."

\section{References}

1. Saba, T. M., W. Alpert, F. A. Blumenstock, G. Evanga, F. Staehler, and E. Cho. 1981. Evaluation of a rapid immunoturbidimetric assay for opsonic fibronectin in surgical and trauma patients. $J$. Lab. Clin. Med. 98:482-491.

2. Yamada, K. M. 1983. Cell surface interactions with extracellular materials. Annu. Rev. Biochem. 52:761-799.

3. Saba, T. M. 1986. Plasma and tissue fibronectin: its role in the pathophysiology of the critically ill septic patient. In Critical Care: State of the Art. Volume 7. B. Chernow and W. C. Shoemaker, editors. Society of Critical Care Medicine, Fullerton, CA. 437-464.

4. Yamada, K. M., and D. W. Kennedy. 1979. Fibroblast cellular and plasma fibronectin are similar but not identical. J. Cell Biol. 80:492-498.

5. Stenman, S., and A. Vaheri. 1978. Distribution of a major connective tissue protein, fibronectin, in normal human tissues. J. Exp. Med. 147:1054-1064.

6. Oh, E., M. Pierschbacher, and E. Ruoslahti. 1981. Deposition of plasma fibronectin in tissues. Proc. Natl. Acad. Sci. USA. 78:32183221.

7. Deno, D. C., T. M. Saba, and E. Lewis. 1983. Kinetics of endogenously labeled plasma fibronectin: incorporation into tissues. $\mathrm{Am}$. J. Physiol. 245:R564-R575.

8. Cohler, L. F., T. M. Saba, E. Lewis, P. A. Vincent, and W. E. Charash. 1987. Plasma fibronectin and lung protein clearance with gram-negative bacteremia: response to fibronectin therapy. J. Appl. Physiol. 63:623-633.

9. Kaplan, J. E., and T. M. Saba. 1976. Humoral deficiency and reticuloendothelial depression after traumatic shock. Am. J. Physiol. 230:7-14.

10. Saba, T. M., and E. Cho. 1979. Reticuloendothelial systemic response to operative trauma as influenced by cryoprecipitate or coldinsoluble globulin therapy. J. Reticuloendothel. Soc. 26:171-186.

11. Saba, T. M., F. A. Blumenstock, D. M. Shah, J. E. Kaplan, E. Cho, W. Scovill, J. Newell, M. Gottlieb, H. Stratton, N. Sedransk, and R. Rahm. 1984. Reversal of fibronectin and opsonic deficiency in patients: a controlled study. Ann. Surg. 299:87-96.

12. Scovill, W. A., T. M. Saba, F. A. Blumenstock, H. Bernard, and S. R. Powers. 1978. Opsonic $\alpha_{2}$ surface binding glycoprotein therapy during sepsis. Ann. Surg. 188:521-529.

13. Scovill, W. A., S. J. Annest, T. M. Saba, F. A. Blumenstock, J. C. Newell, H. H. Stratton, and S. R. Powers. 1979. Cardiovascular hemodynamics after opsonic $\alpha_{2} \mathrm{SB}$ glycoprotein therapy in injured patients. Surgery. 86:284-293.
14. Saba, T. M., F. A. Blumenstock, D. M. Shah, J. E. Kaplan, E. Cho, W. Scovill, J. Newell, M. Gottlieb, H. Stratton, N. Sedransk, and R. Rahm. 1986. Reversal of opsonic deficiency of purified human plasma fibronectin. Am. J. Med. 80:229-240.

15. Wolfe, F. 1984. Tracers in Metabolic Research. Alan R. Liss, Inc., New York. 264-268.

16. Millard, B. I. 1979. Quantitative Mass Spectrometry. Heyden and Sons, Ltd., Philadelphia, PA. 60-89.

17. Amrani, D. L., D. Mauzy-Melitz, and M. W. Mosesson. 1986. Effect of hepatocyte-stimulating factor and glucocorticoids on plasma fibronectin levels. Biochem. J. 238:365-371.

18. Kaplan, J. E., F. A. Blumenstock, and T. M. Saba. 1979. A radial immunodiffusion method for the measurement of rat fibrinogen and fibrin degradation products. Vox Sang. 36:65-71.

19. Tamkun, J., and R. O. Hynes. 1983. Plasma fibronectin is synthesized and secreted by hepatocytes. J. Biol. Chem. 258:46414647.

20. Owens, M. R., and C. D. Cimino. 1982. Synthesis of fibronectin by isolated perfused rat liver. Blood. 59:1305-1309.

21. Stein, T. P., M. J. Leskew, and H. W. Wallace. 1976. Equilibration of ${ }^{15} \mathrm{~N}$-labeled amino compounds in man. Am. J. Physiol. 236:1326-1336.

22. Wu, H., and C. W. Bishop. 1959. Pattern of ${ }^{15} \mathrm{~N}$ excretion in man following administration of ${ }^{15} \mathrm{~N}$-labeled glycine. J. Appl. Physiol. 14:1-4.

23. Dixon, W. J. 1985. BMDP Statistical Software Manual. University of California Press, Berkeley, CA.

24. Ralston, M. L., R. I. Jennrich, D. F. Samson, and F. K. Uno. 1979. Fitting models defined by differential equations. Technical Report No. 70. BMDP Statistical Software Inc., Los Angeles, CA.

25. Yudkoff, M., I. Nissim, W. Michellis, and R. Polin. 1987. Albumin synthesis in premature infants: determination of turnover with [ $\left.{ }^{15} \mathrm{~N}\right] \mathrm{glycine}$. Pediatr. Res. 21:49-53.

26. Richards, W. O., W. A. Scovill, and B. Shin. 1983. Opsonic fibronectin deficiency in patients with intra-abdominal infection. Surgery. 94:210-217.

27. Lanser, M. E., T. M. Saba, and W. A. Scovill. 1980. Opsonic glycoprotein (plasma fibronectin) levels after burn injury: relationship to extent of burn and development of sepsis. Ann. Surg. 192:776-782.

28. Richards, P. S., and T. M. Saba. 1985. Effect of endotoxin and Kupffer cell activity. Hepatology. 5:32-37.

29. Graninger, W., K. Pirich, K. Derfler, and W. Waldhausl. 1985. Plasma fibronectin and thyroid function. J. Clin. Pathol. 38:64-67.

30. Azuma, H., T. Takeichi, T. Ohara, and A. Shirakami. 1987. Metabolism of plasma fibronectin in rabbits with experimental hyperthyroidism and hypothyroidism. Metab. Clin. Exp. 36:777-780.

31. Dillon, B. C., T. M. Saba, E. Cho, and E. Lewis. 1982. Opsonic fibronectin deficiency in the etiology of starvation induced reticuloendothelial phagocytic dysfunction. Exp. Mol. Pathol. 36:177-192.

32. Howard, L., B. Dillon, T. M. Saba, S. Hofmann, and E. Cho. 1984. Decreased plasma fibronectin during starvation in man. J. Parenter. Enter. Nutr. 8:239-244.

33. Sherman, L. A., and J. Lee. 1982. Fibronectin turnover in normal animals and during intravascular coagulation. Blood. 60:558563.

34. Mosher, D. F., and E. M. Williams. 1978. Fibronectin concentration is decreased in plasma of severely ill patients with disseminated intravascular coagulation. J. Lab. Clin. Med. 91:729-735.

35. Kiener, J. L., T. M. Saba, E. Cho, and F. A. Blumenstock. 1986. Clearance and distribution of plasma fibronectin in septic rats: relationship to synthetic rate. Am. J. Physiol. 251:R724-R734.

36. Engvall, E., and E. Ruoslahti. 1977. Binding of soluble fibroblast surface protein, fibronectin, to collagen. Int. J. Cancer. 20:1-5.

37. Gersovitz, M., H. N. Munro, J. Udall, and V. R. Young. 1980. Albumin synthesis in young and elderly subjects using a new stable isotope methodology: response to level of protein intake. Metab. Clin. Exp. 29:1075-1086. 
38. Stein, T. P., M. J. Leskin, and H. W. Wallace. 1978. Measurement of half-life of human plasma fibrinogen. Am. J. Physiol. 234:E504-E510.

39. Bowersox, J. C., and R. L. Scott. 1985. Plasma fibronectin metabolism during hemorrhagic shock and starvation. J. Surg. Res. 39:445-453.

40. Pussell, B. A., P. W. Peake, M. A. Brown, and J. A. Charlesworth. 1985. Human fibronectin metabolism. J. Clin. Invest. 76:143148.

41. Koj, A. 1985. Cytokines regulating acute inflammation and synthesis of acute phase proteins. Blut. 51:267-274.

42. Castell, J. V., M. J. Gomez-Lechon, M. David, T. Hirano, T. Kishimoto, and P. C. Heinrich. 1988. Recombinant human interleu- kin-6 (IL-6/BSF-2/HSF) regulates the synthesis of acute phase proteins in human hepatocytes. FEBS (Fed. Eur. Biochem. Soc.) Lett. 232:347-350.

43. DeCamp, M., and R. H. Demling. 1988. Posttraumatic multisystem organ failure. JAMA (J. Am. Med. Assoc.) 260:530-534.

44. Saba, T. M., and E. Jaffe. 1980. Plasma fibronectin (opsonic glycoprotein): its synthesis by vascular endothelial cells and role in cardiopulmonary integrity after trauma as related to reticuloendothelial function. Am. J. Med. 68:577-594.

45. Polin, R. A., M. C. Yoder, S. D. Douglas, W. McNelis, I. Nissim, and M. Yudkoff. 1989. Fibronectin turnover in the premature

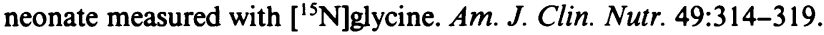

